\section{The International System of Units is getting a Makeover}

\section{by lan Mills and Roberto Marquardt}

M etrology, the science of measurement, is part of the essential but largely hidden infrastructure of the modern world. We need it for high-technology manufacturing, human health and safety, the protection of the environment, global climate studies, information transfer and the basic science that underpins all these. Highly accurate measurements are no longer the preserve of only the physical sciences and engineering. The International System of Units, the SI (Système International d'unités), provides the internationally agreed means by which we make such measurements.

At a meeting of the General Conference on Weights and Measures (CGPM) held in Paris on 16 November 2018 a new and revised SI was approved, adapted for the 21 st century. This note is to bring these changes to the attention of readers of Chemistry International. These changes will be implemented on 20 May 2019, the next "World Metrology Day" and the day anniversary on which the Convention of the Metre was first signed in 1875. Of particular interest to chemists are the changes in the definition of the SI units kilogram and mole.

In this context the well-known opening sentence of Jane Austen's novel Pride and Prejudice may be adapted to read: "It is a truth universally acknowledged that a single man in possession of a good fortune, must be in want of a good set of units." In the last four words of this sentence Jane Austen wrote "must be in want of a wife," but we have substituted "in want of a good set of units." Perhaps we need both!

The following is a brief summary of the changes that are being adopted in this new SI.

1. Each of the seven base units of the SI (second, metre, kilogram, ampere, kelvin, mole, and candela) is defined by an agreed reference that has to be readily available and easily realized experimentally by anyone anywhere at any time with sufficient precision (sufficiently low uncertainty) for our needs. Four of the seven base units, the kilogram, ampere, kelvin and mole, have revised definitions in the new $\mathrm{SI}$ as described below. The definitions of the second, metre and candela remain unchanged.

2. All of the new definitions will in future be expressed in terms of seven 'defining constants' which

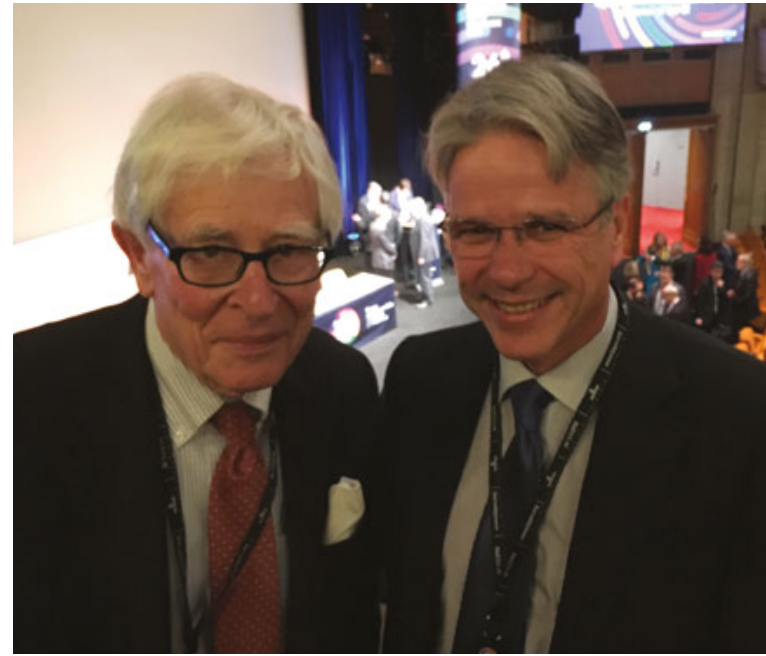

Ian Mills and Roberto Marquardt at the meeting of the CGPM in Paris on 16 November 2018

are believed to fulfil these requirements and are summarized below. The numerical values of these seven constants when expressed in SI units provides the definition of all SI units, both base and derived units.

3. The revised definition of the kilogram is chosen to fix the numerical value of the Planck constant $h$, that of the ampere to fix the numerical value of the elementary charge $e$, that of the kelvin to fix the numerical value of the Boltzmann constant $k\left(k_{\mathrm{B}}\right)$, and that of the mole to fix the numerical value of the Avogadro constant $N_{\mathrm{A}}(L)$, all these numerical values being expressed in terms of the corresponding SI unit. The definitions of the remaining three base units, the second, the metre, and the candela fix the numerical value of the caesium hyperfine splitting $\Delta v_{\mathrm{Cs}}$, the speed of light in vacuum $c$, and the luminous intensity of the specified source $I_{v}$ just as they do at present.

4. The values of the seven defining constants listed in Table 1 below are chosen to be consistent with the best experimental values at the time of adopting the new definitions, to preserve continuity.

5. To summarize, the International System of Units, the $\mathrm{SI}$, is the system of units in which:

- the unperturbed ground state hyperfine transition frequency of the caesium 133 atom $\Delta v_{\text {Cs }}$ is exactly $9192631770 \mathrm{~Hz}$,

- the speed of light in vacuum $c$ is exactly $299792458 \mathrm{~m} / \mathrm{s}$,

- the Planck constant $h$ is exactly $6.62607015 \times 10^{-34} \mathrm{~J} \mathrm{~s}$,

- the elementary charge $e$ is exactly $1.602176634 \times 10^{-19} \mathrm{C}$,

- the Boltzmann constant $k$ is exactly $1.380649 \times 10^{-23} \mathrm{~J} / \mathrm{K}$, 
- the Avogadro constant $N_{A}$ is exactly

$6.02214076 \times 10^{23} \mathrm{~mol}^{-1}$,

- the luminous efficacy $K_{c d}$ of monochromatic radiation of frequency $540 \times 10^{12} \mathrm{~Hz}$ is exactly $683 \mathrm{~lm} / \mathrm{W}$.

where the hertz, joule, coulomb, lumen, and watt, with unit symbols $\mathrm{Hz}, \mathrm{J}, \mathrm{C}, \mathrm{Im}$, and $\mathrm{W}$, respectively, are related to the units second, metre, kilogram, ampere, kelvin, mole, and candela, with unit symbols $\mathrm{s}, \mathrm{m}, \mathrm{kg}, \mathrm{A}, \mathrm{K}, \mathrm{mol}$, and $\mathrm{cd}$, respectively, according to $\mathrm{Hz}=\mathrm{s}^{-1}, \mathrm{~J}=\mathrm{m}^{2} \mathrm{~kg} \mathrm{~s}^{-2}$, $C=A s, I m=c d s r$, and $W=m^{2} \mathrm{~kg} \mathrm{~s}^{-3}$.

\begin{tabular}{|c|c|c|c|}
\hline Defining constant & Symbol & Numerical value & Unit \\
\hline $\begin{array}{l}\text { hyperfine transition } \\
\text { frequency of caesium }\end{array}$ & $\Delta v_{\mathrm{CS}}$ & 9192631770 & $\mathrm{~Hz}$ \\
\hline $\begin{array}{l}\text { speed of light in } \\
\text { vacuum }\end{array}$ & c & 299792458 & $\mathrm{~m} \mathrm{~s}^{-1}$ \\
\hline Planck constant & $h$ & $6.62607015 \times 10^{-34}$ & $\mathrm{Js}$ \\
\hline elementary charge & e & $1.602176634 \times 10^{-19}$ & C \\
\hline Boltzmann constant & k & $1.380649 \times 10^{-23}$ & $\mathrm{~J} \mathrm{~K}^{-1}$ \\
\hline Avogadro constant & $N_{\mathrm{A}}$ & $6.02214076 \times 10^{23}$ & $\mathrm{~mol}^{-1}$ \\
\hline luminous efficacy & $K_{c d}$ & 683 & $\operatorname{Im~} \mathrm{W}^{-1}$ \\
\hline
\end{tabular}

Table 1: The seven defining constants of the SI, and the seven corresponding symbols, numerical values, and units

The numerical values of the seven defining constants have zero uncertainty. They are summarized in Table 1 on this page. Definitions based on defining constants are called explicit-constant definitions; they are based on the fundamental constants of nature, in contrast to explicit-unit definitions which are based on particular experimental procedures.

Defining a unit by specifying the numerical value of a fundamental constant may be understood as follows. The value of any quantity $Q$ may always be represented as the product of its numerical value $\{Q\}$ and a unit $[Q]$, so that we may write $Q=\{Q\}[Q]$ (for example $c=299792458 \mathrm{~m} / \mathrm{s}$ for the speed of light in vacuum). If the quantity $Q$ is itself a unit that we wish to define, this may be done either by specifying some convenient reference (such as the length of the prototype metre bar that was used to define the SI unit of length prior to 1980) or by specifying the numerical value $\{Q\}$ when expressed in terms of the desired $S I$ unit (such as the numerical value of the speed of light 299792458 expressed in the unit m/s used to define the metre since 1980). It is this second method of using defining constants that is now being adopted for all the seven base units of the SI.

For chemists, the definition of the mole has an important conceptual consequence. It is namely equivalent to stating that "One mole contains exactly $6.02214076 \times 10^{23}$ elementary entities," quite in the spirit of William Shakespeare in As You Like It: "It is as easy to count atomies as to resolve the propositions of a lover." In addition, the exact and fixed number of elementary entities defining a mole finally receives the name that has been used for it for decades without proper definition: the Avogadro number.

In general, the changes associated with the new SI will lead to reduced uncertainties in our knowledge of most of the fundamental constants of physics and chemistry in the new SI.

The changes in the new SI will strengthen the philosophical foundation of our system of units in relation to our present understanding of theoretical and quantum physics. However, they will not affect the daily work in the laboratory in any sizeable manner.

What are the direct consequences of these changes to a chemist working in the laboratory? For example, the equation

$$
\mathrm{C}_{2} \mathrm{H}_{4} \mathrm{O}=\mathrm{CH}_{4}+\mathrm{CO}
$$

has the meaning that one mole of oxirane $\left(\mathrm{C}_{2} \mathrm{H}_{4} \mathrm{O}\right)$ decomposes to yield one mole of methane $\left(\mathrm{CH}_{4}\right)$ and one mole of carbon monoxide (CO). The new definition of

\begin{tabular}{|lll|lll|}
\hline constant & current SI & new SI & constant & current SI & new SI \\
\hline$m(K)$ & 0.0 & 5.0 & $a$ & 0.068 & 0.068 \\
\hline$h$ & 5.0 & 0.0 & $K_{\mathrm{J}}$ & 2.5 & 0.0 \\
\hline$e$ & 2.5 & 0.0 & $R_{\mathrm{K}}$ & 0.068 & 0.0 \\
\hline$K$ & 170 & 0.0 & $\mu_{\mathrm{O}}$ & 0.0 & 0.068 \\
\hline$N_{\mathrm{A}}$ & 5.0 & 0.0 & $\varepsilon_{\mathrm{o}}$ & 0.0 & 0.068 \\
\hline$R$ & 170 & 0.0 & $Z_{\mathrm{o}}$ & 0.0 & 0.068 \\
\hline$F$ & 2.5 & 0.0 & $N_{\mathrm{A}} h$ & 0.14 & 0.0 \\
\hline$\sigma$ & 700 & 0.0 & $\mathrm{~J} \leftrightarrow \mathrm{kg}$ & 0.0 & 0.0 \\
\hline$m_{\mathrm{e}}$ & 5.0 & 0.14 & $\mathrm{~J} \leftrightarrow \mathrm{m}^{-1}$ & 5.0 & 0.0 \\
\hline$m_{\mathrm{u}}$ & 5.0 & 0.14 & $\mathrm{~J} \leftrightarrow \mathrm{Hz}$ & 5.0 & 0.0 \\
\hline$m\left({ }^{12} \mathrm{C}\right)$ & 5.0 & 0.14 & $\mathrm{~J} \leftrightarrow \mathrm{K}$ & 170 & 0.0 \\
\hline$\left.{ }^{12} \mathrm{C}\right)$ & 0.0 & 0.14 & $\mathrm{~J} \leftrightarrow \mathrm{eV}$ & 2.5 & 0.0 \\
\hline
\end{tabular}

Table 2: Relative standard uncertainties for a selection of fundamental constants in the current $\mathrm{SI}$ and the new SI, multiplied by $10^{8}$ (i.e. in parts per hundred million) Note: in this table the symbols denote the following constants: $R$ : the molar gas constant; $F$ : the Faraday constant; $\sigma$ : the Stefan-Boltzmann constant; $m_{\mathrm{e}}$ : the electron mass; $m_{\mathrm{u}}$ : the unified atomic mass constant; $m\left({ }^{12} \mathrm{C}\right)$ : the mass of a carbon 12 atom; $M\left({ }^{12} \mathrm{C}\right)$ : the molar mass of carbon 12; $a$ : the fine structure constant; $K_{\mathrm{J}}$ and $R_{\mathrm{K}}$ : the Josephson and von Klitzing constants; $\mu_{0}$ and $\varepsilon_{0}$ the magnetic and electric constants; $Z_{0}$ : the impedance of vacuum; $N_{\mathrm{A}} h$ : the molar Planck constant; and $U_{\mathrm{a}} \leftrightarrow U_{\mathrm{b}}$ : the conversion factor between unit $a$ and unit $b$. 
the mole will not change this meaning. A chemist in the laboratory will continue to determine the amount of a chemical entity $\mathrm{B}, n(\mathrm{~B})$, by weighing the corresponding mass $m(B)$ and setting $n(B)=m(B) / M(B)$, where $M(B)$ is the molar mass of $B$. He/she will continue to state that $44.053 \mathrm{~g}$ of oxirane decomposes to yield $16.043 \mathrm{~g}$ methane and $28.010 \mathrm{~g}$ carbon monoxide. Truly, the molar mass $M(B)=M_{r}(B) M_{u}$ will acquire an uncertainty component of less than 1 part in $10^{9}$ due to the new uncertainty of the molar mass constant $M_{u}=$ $M\left({ }^{12} \mathrm{C}\right) / 12$ (see Table 2; the relative molar mass $M_{r}(B)$ of any atom $B$ is unchanged in the new $\mathrm{SI}$ ). However, balances in chemistry laboratories will continue to yield masses (e.g. in the SI unit $\mathrm{kg}$ ) with uncertainties that far exceed the uncertainty of the molar mass of any given chemical entity by orders of magnitude, so that the change in the new definition of the mole will never influence the result of the determinations of amount of substance in practice.

\section{References}

Marquardt, R., Meija, J., Mester, Z., et al. (2017). A critical review of the proposed definitions of fundamental chemical quantities and their impact on chemical communities (IUPAC Technical Report). Pure Appl. Chem., 89, pp. 951981; https://doi.org/10.1515/pac-2016-0808

Marquardt, R., Meija, J., Mester, Z., et al. (2018). Definition of the mole (IUPAC Recommendation 2017). Pure Appl. Chem., 90, pp. 175-180; https://doi.org/10.1515/pac-20170106

Mills, I.M, Mohr, P.J., Quinn, T.J., Taylor, B.N. and Williams, E. R. (2006). Redefinition of the kilogram, ampere, kelvin and mole: a proposed approach to implementing CIPM recommendation 1 (Cl-2005). Metrologia, 43, pp. 227246; https://doi.org/10.1088/0026-1394/43/3/006

Mills, I.M., Mohr, P.J., Quinn, T.J., Taylor, B.N. and Williams, E.R. (2011). Adapting the International System of Units to the twenty-first century. Philosophical Transactions of the Royal Society A: Mathematical, Physical and Engineering Sciences 369, pp. 3907-3924; https://doi.org/10.1098/ rsta. 2011.0180

Ullrich, J. Why we need the SI redefinition. EURAMET short videos (13 June 2018), https://www.euramet. org/?news $=40: 678$

Bureau International des Poids et Mesures (BIPM), On the Future Revision of the SI (International System of Units or Système International d'Unités), https://www.bipm. org/en/measurement-units/rev-si/

MIls, I.M., The Kilogram in the New SI-part II Explicit Constant Definitions for the Kilogram and for the Mole (2011) Chem. Int. 33(5) pp. 12-15; https://doi.org/10.1515/ ci.2011.33.5.12

lan Mills <i.m.mills@reading.ac.uk> is emeritus professor of chemistry at the University of Reading, UK and an elected Fellow of the Royal Society. He was the President of Consultative Committee on Units of the International Bureau of Weights and Measures (BIPM) and retired from this role in 2013 after 18 vears of service. In IUPAC, he served as chair of the Commission on Physicochemical Symbols, Terminology, and Units (Commission I.1), and also as chair of the Interdivisional Committee on Nomenclature and Symbols (IDCNS, preceding the current ICTNS) until 1999.

Roberto Marquardt <roberto.marquardt@unistra.fr> was President of the Physical and Biophysical Chemistry Division of IUPAC in 2014-15. 


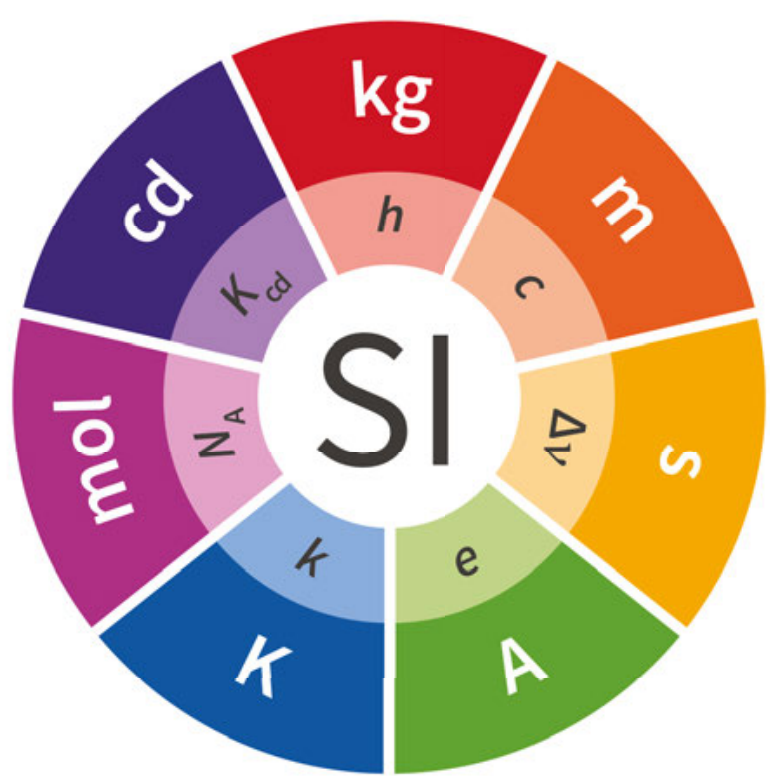

The Seven Base Units of the new SI are defined as in the table below*

Quantity SI unit

time The second, symbol s, is the SI unit of time. It is defined by taking the fixed numerical value of the caesium frequency $\Delta v_{\mathrm{Cs}}$, the unperturbed ground-state hyperfine transition frequency of the caesium 133 atom, to be 9192631770 when expressed in the unit $\mathrm{Hz}$, which is equal to $\mathrm{s}^{-1}$.

length The metre, symbol $\mathrm{m}$, is the $\mathrm{SI}$ unit of length. It is defined by taking the fixed numerical value of the speed of light in vacuum $c$ to be 299792458 when expressed in the unit $\mathrm{m} \mathrm{s}^{-1}$, where the second is defined in terms of the caesium frequency $\Delta v_{\mathrm{cs}}$.

mass

The kilogram, symbol $\mathrm{kg}$, is the SI unit of mass. It is defined by taking the fixed numerical value of the Planck constant $h$ to be $6.62607015 \times 10^{-34}$ when expressed in the unit $\mathrm{J} \mathrm{s}$, which is equal to $\mathrm{kg} \mathrm{m}^{2} \mathrm{~s}^{-1}$, where the metre and the second are defined in terms of $c$ and $\Delta v_{c s}$.

electric The ampere, symbol A, is the SI unit of electric current. It is defined by taking the fixed current numerical value of the elementary charge e to be $1.602176634 \times 10^{-19}$ when expressed in the unit $\mathrm{C}$, which is equal to $\mathrm{A} s$, where the second is defined in terms of $\Delta v_{\mathrm{Cs}}$.

thermodynamic temperature

The kelvin, symbol $\mathrm{K}$, is the $\mathrm{SI}$ unit of thermodynamic temperature. It is defined by taking the fixed numerical value of the Boltzmann constant $k$ to be $1.380649 \times 10^{-23}$ when expressed in the unit $\mathrm{J} \mathrm{K}^{-1}$, which is equal to $\mathrm{kg} \mathrm{m}^{2} \mathrm{~s}^{-2} \mathrm{~K}^{-1}$, where the kilogram, metre and second are defined in terms of $h, c$ and $\Delta v_{\text {Cs }}$.

amount of substance

The mole, symbol mol, is the $\mathrm{SI}$ unit of amount of substance. One mole contains exactly $6.02214076 \times 10^{23}$ elementary entities. This number is the fixed numerical value of the Avogadro constant, $N_{\mathrm{A}}$, when expressed in the unit $\mathrm{mol}^{-1}$ and is called the Avogadro number. The amount of substance, symbol $n$, of a system is a measure of the number of specified elementary entities. An elementary entity may be an atom, a molecule, an ion, an electron, any other particle or specified group of particles.

luminous intensity

The candela, symbol cd, is the SI unit of luminous intensity in a given direction. It is defined by taking the fixed numerical value of the luminous efficacy of monochromatic radiation of frequency $540 \times 10^{12} \mathrm{~Hz}, K_{\mathrm{cd}}$, to be 683 when expressed in the unit Im W'-1, which is equal to $c d s r W^{-1}$, or $c d \mathrm{sr} \mathrm{kg}^{-1} \mathrm{~m}^{-2} \mathrm{~s}^{3}$, where the kilogram, metre and second are defined in terms of $h, c$ and $\Delta v_{\mathrm{cs}}$.

${ }^{*}$ Table reproduced from https://www.bipm.org/utils/en/pdf/si-revised-brochure/Draft-SI-Brochure-2018.pdf (accessed 31 Oct 2018). The concept of base units and derived units was used to define the SI until 2018. These categories, although not essential in the new SI, are maintained in view of their convenience and widespread use. 